\title{
A new era in the management of type 2 diabetes: is cardioprotection at long
}

\section{last a reality!}

Xavier Rossello, MD ${ }^{1}$, Derek M. Yellon, PhD, DSc, FRCP ${ }^{1,2}$

\author{
${ }^{1}$ The Hatter Cardiovascular Institute, University College London, London, United Kingdom \\ ${ }^{2}$ NIHR UCLH Biomedical Research Centre, University College London Hospital \& Medical School, \\ London, United Kingdom
}

\section{Address for correspondence:}

Derek M. Yellon, The Hatter Cardiovascular Institute, University College London,

67 Chenies Mews, London WC1E 6HX 2, United Kingdom. Email: d.yellon@ucl.ac.uk

\section{Conflicts of interest}

None declared.

Funding sources. This work was supported by a proportion of funding from the Department of Health's NIHR Biomedical Research Centres funding scheme. Dr Rossello has received support from the Fundacion Alfonso Martin Escudero fellowship grant.

Word count: 1516 


\begin{abstract}
The EMPA-REG OUTCOME and the LEADER trials have revealed a new era in the management of type 2 diabetes. The SGLT2 inhibitor empagliflozin demonstrated a lower rate of the primary composite outcome of death from cardiovascular causes, nonfatal myocardial infarction, or nonfatal stroke compared to placebo. Liraglutide, a GLP-1 analogue, succeeded to demonstrate reduction on a composite outcome including first occurrence of cardiovascular death, nonfatal myocardial infarction or non-fatal stroke. These two medications act through different mechanisms and has consequently shown different patterns of cardiovascular benefit. In one hand, empagliflozin showed an earlier effect compared to those observed using liraglutide. In the other hand, the difference between empagliflozin and placebo was driven by a significant reduction in death from cardiovascular causes, with and striking disconnect showing no significant between-group difference in the risk of myocardial infarction or stroke. In contrast, liraglutide reduced consistently all components of the composite endpoint. Based on the different temporal pattern of achieving clinical benefit one might flirt with the idea that liraglutide seems to provide a chronic "protection" that better fits in a longer metabolic effect with an impact in the progression of atherosclerosis, whilst empagliflozin provides an acute effect compatible with an immediate hemodynamic action. After years going from "bench to bedside" in order to discover the holy grail of cardioprotection, these 2 new studies suggest that we may have reached this state and it is time to go from "bed back to bench side" to understand the mechanisms of this potential paradigm shift.
\end{abstract}

Key words: cardioprotection; diabetes; SGLT2 inhibitor; GLP-1 analogue 
Type 2 diabetes mellitus (T2DM) is expected to affect 552 million people worldwide by 2030 . T2DM patients have two- to three-fold greater risk of presenting cardiovascular events compared with nondiabetics. Furthermore, as many as $80 \%$ of T2DM individuals will die from cardiovascular complications, such as myocardial infarction, stroke and peripheral vascular disease.

The link between diabetes and cardiovascular was historically thought to be a solely atherosclerosis driven process, although this concept has lately evolved to a more complex interplay between several factors affected by T2DM, such as vulnerable blood constituents and vulnerable myocardium, in addition to the well-known vulnerable plaque. Hence, T2DM has an impact not only in the rates of cardiovascular events, but also in the damage caused by them.

Unlike the microvascular complications associated with T2DM, evidence that glucose lowering is associated with macrovascular benefits is less certain. However, antidiabetic drug effects have also been studied beyond their glucose-lowering capacity. In the setting of ischemia-reperfusion injury (IRI), known as the additional myocardial damage that negatively impacts on myocardial infarct size due to the process of restoring blood flow to the ischemic myocardium[1], antidiabetics have shown conflicting results. On one hand, there is modest evidence suggesting that sulfonylureas may further impair IRI damage in diabetic patients, whilst on the other hand, several preclinical and clinical proof of concept studies have suggested that insulin, metformin, dipeptidyl peptidase-4 (DPP-4) inhibitors and glucagon-like peptide-1 (GLP-1) analogues confer myocardial protection against IRI[1,2].

However we are fortunate that new agents may provide the answer to beneficial cardiovascular outcomes; these being the GLP-1 analogues and the sodium-glucose cotransporter 2 (SGLT2) inhibitors.

GLP-1 is an incretin hormone, released from the intestine in response to enteral nutrition, that reduces postprandial hyperglycemia by stimulating insulin secretion from $\beta$ cells in the pancreas and inhibiting glucagon secretion from pancreatic $\alpha$ cells, and induces weight loss by acting on brain appetite-control centers. The active form, GLP-1(7-36), which is rapidly degraded by the enzyme DPPIV, binds GLP-1 receptor, which is expressed not only in pancreatic islet cells, but also in the kidney, lung, brain, gastrointestinal tract and heart. Research has manipulated the biology of the entero-insular axis producing both injectable GLP-1 agonists that are used as an exogenous source of GLP-1 and oral 
DPP-4 inhibitors that shield the endogenous peptide from degradation[3]. Findings from our laboratory demonstrated for the first time that the administration of either GLP-1 native peptide or the DPP-4 inhibitor protects against myocardial IRI in the isolated rat heart model through a mechanism not driven by the stimulation of insulin secretion, but by the activation of intracellular prosurvival kinases cascades[4]. Subsequently, Lonborg et al showed that the infusion of exenatide, a GLP-1analogue, prior to primary percutaneous coronary intervention reduces myocardial infarct size patients presenting with ST-segment elevation myocardial infarction[5].

SGLT2 inhibition, reduces rates of hyperglycemia by decreasing glucose reabsorption in the renal proximal tubule, thereby increasing urinary glucose excretion[6]. SGLT2 inhibition also decreases sodium reabsorption, exerting both a diuretic and natriuretic effect that impact to decrease blood pressure and diminish extracellular volume. Overall, SGLT2 inhibitors provides both metabolic and hemodynamic benefits.

The New England Journal of Medicine has published a consecutive series of randomized clinical trials (RCTs) with regard to the effect of antidiabetic drugs on cardiovascular outcomes in T2DM patients. In 2013, two cardiovascular outcome trials examining DPP-4 inhibitors; the Saxagliptin Assessment of Vascular Outcomes Recorded in Patients with Diabetes Mellitus-Thrombolysis in Myocardial Infarction 53 (SAVOR-TIMI 53) trial[7] and the Examination of Cardiovascular Outcomes with Alogliptin versus Standard of Care (EXAMINE) trial[8], showed that these agents did not have an impact on cardiovascular outcomes, but raised safety concerns regarding a potential increased risk of hospitalization for heart failure. In 2015, a third trial testing a DPP-4 inhibitor, the trial Evaluating Cardiovascular Outcomes with Sitagliptin (TECOS)[9], found that the addition of sitagliptin to usual care among patients with glycemic equipoise does not affect rates of major atherosclerotic cardiovascular events, including changes in rates of hospitalization for heart failure[9]. In 2015, the Evaluation of Lixisenatide in Acute Coronary Syndrome (ELIXA) trial[10] reported that another incretin-based therapy, a GLP-1 receptor analogue, did not significantly affect the rate of major cardiovascular events or other serious adverse events in T2DM with a recent acute coronary syndrome.

After this wave of studies, two recent outcome trials have marked a turning point in cardiovascular medicine. The Empagliflozin Cardiovascular Outcome Event Trial in Type 2 Diabetes Mellitus Patients 
(EMPA-REG OUTCOME)[11] trial and the Liraglutide Effect and Action in Diabetes: Evaluation of Cardiovascular Outcome Results - A Long Term Evaluation (LEADER) trial[12] have revealed a new era in the management of T2DM, demonstrating cardiovascular benefit rather than just lack of harm. These two medications act through different mechanisms and has consequently shown different patterns of cardiovascular benefit.

When the SGLT2 inhibitor empagliflozin was administered alongside the standard care among patients with T2DM at high risk for cardiovascular, this group showed a lower rate of the primary composite outcome of death from cardiovascular causes, nonfatal myocardial infarction, or nonfatal stroke compared to placebo[11]. In the same vein, liraglutide, a GLP-1 analogue, succeeded to demonstrate reduction on a composite outcome including first occurrence of cardiovascular death, nonfatal myocardial infarction or non-fatal stroke[12]. Despite being powered as a noninferiority study, the Trial to Evaluate Cardiovascular and Other Long-term Outcomes with Semaglutide in Subjects with Type 2 Diabetes (SUSTAIN-6) have also demonstrated that semaglutide (another GLP-1 analogue) reduces the risk of cardiovascular death, nonfatal myocardial infarction and nonfatal stroke [13]. We intend to focus on the two studies designed to demonstrate superiority, being representative of two distinct class of anti-diabetic drugs with cardiovascular effects.

In this regard although both empagliflozin [11] and liraglutide[12] have shown cardiovascular benefit, they have demonstrated a different temporal and effect pattern. First, the separation between treatment and placebo time-to-event curves occurred earlier in the empagliflozin trial, whilst liraglutide appears to have a more constant and late effect. Second, the difference between empagliflozin and placebo was driven by a significant reduction in death from cardiovascular causes, with and striking disconnect showing no significant between-group difference in the risk of myocardial infarction or stroke. In contrast, liraglutide reduced consistently all components of the composite endpoint. Although these trials were designated to evaluate the effect of these drugs on cardiovascular outcomes, we might speculate on the mechanisms behind the observed benefits.

It is well known that these two drugs have both a metabolic e.g. weight loss effect and a haemodynamic effect e.g. blood pressure lowering effect all of which may help with overall myocardial benefit. There are no available tools to elucidate how much glucose control contribute to provide 
cardiovascular benefits as it does with microvascular complications[14]. However, based on the different temporal pattern of achieving clinical benefit one might flirt with the idea that liraglutide seems to provide a chronic "protection" that better fits in a longer metabolic effect with an impact in the progression of atherosclerosis, whilst empagliflozin provides an effect manifested in the short-term compatible with an immediate hemodynamic action, such as decrease in blood pressure or increase glucagon an inotrope that might be beneficial in heart failure. Imagine what the 2 together could achieve? In addition the potential relevance of glucagon should be mentioned. It has been suggested that the inhibition of this peptide could diminish metabolic efficiency (as seen with a GLP-1 analogue) however the SGLT2 inhibitors have been shown to increase the levels of this peptide which could neutralise any such effect.

Remarkably we might be at the birth of a whole new era in cardioprotection based upon the extraordinary results of these two ground-breaking trials. It could be argued that the SGLT2 inhibitor fits perfectly the profile of a potential cardioprotective intervention. Its reduction of cardiovascular mortality without decrease in neither nonfatal myocardial infarction nor nonfatal stroke suggests that the beneficial effect of empagliflozin lies in the survival improvement among T2DM patients experiencing a cardiovascular event rather than in the prevention of atherosclerotic events. Paradoxically, SGLT2 is not known to be expressed in cardiomyocytes, so after many years investigating myocardial signalling pathways cardioprotection might finally arrive through a completely different mechanism. In the case of incretin-based therapies, GLP-1 agonists are a promising therapy to be used to reduce IRI. GLP-1 agonists are an exogenous source of the molecule with improved pharmacokinetic properties that increases pharmacologic levels of GLP-1, whilst DPP-4 inhibitors "only" shield the endogenous peptide from degradation. Consequently, the level of biologically active GLP-1 made available by oral DPP-4 inhibitor therapy is typically three- to fivefold less than that provided by GLP-1 receptor agonists, potentially making a difference to obtain clinical benefit, maybe also through IRI protection.

After years going from "bench to bedside" in order to discover the holy grail of cardioprotection, these 2 new studies suggest that we may have reached this state and it is time to go from "bed back to bench side" to understand the mechanisms of this potential paradigm shift. Therefore if these new anti- 
diabetic agents are telling us that it is possible to protect the myocardium; we need to pay attention and exploit this outcome to our advantage. 


\section{References}

[1] X. Rossello, D.M. Yellon, A critical review on the translational journey of cardioprotective therapies!, Int. J. Cardiol. 220 (2016) 176-184. doi:10.1016/j.ijcard.2016.06.131.

[2] K. Chinda, S. Palee, S. Surinkaew, M. Phornphutkul, S. Chattipakorn, N. Chattipakorn, Cardioprotective effect of dipeptidyl peptidase-4 inhibitor during ischemia-reperfusion injury, Int. J. Cardiol. 167 (2016) 451-457. doi:10.1016/j.ijcard.2012.01.011.

[3] G. Savarese, C. D’Amore, M. Federici, F. De Martino, S. Dellegrottaglie, C. Marciano, et al., Effects of Dipeptidyl Peptidase 4 Inhibitors and Sodium-Glucose Linked coTransporter-2 Inhibitors on cardiovascular events in patients with type 2 diabetes mellitus: A meta-analysis, Int. J. Cardiol. 220 (2016) 595-601. doi:10.1016/j.ijcard.2016.06.208.

[4] A.K. Bose, M.M. Mocanu, R.D. Carr, C.L. Brand, D.M. Yellon, Glucagon-like peptide 1 can directly protect the heart against ischemia/reperfusion injury., Diabetes. 54 (2005) 146-51. http://www.ncbi.nlm.nih.gov/pubmed/15616022 (accessed June 6, 2016).

[5] J. Lønborg, N. Vejlstrup, H. Kelbæk, H.E. Bøtker, W.Y. Kim, A.B. Mathiasen, et al., Exenatide reduces reperfusion injury in patients with ST-segment elevation myocardial infarction., Eur. Heart J. 33 (2012) 1491-9. doi:10.1093/eurheartj/ehr309.

[6] R.K. Ghosh, D. Bandyopadhyay, A. Hajra, M. Biswas, A. Gupta, Cardiovascular outcomes of sodium-glucose cotransporter 2 inhibitors: A comprehensive review of clinical and preclinical studies, Int. J. Cardiol. 212 (2016) 29-36. doi:10.1016/j.ijcard.2016.02.134.

[7] B.M. Scirica, D.L. Bhatt, E. Braunwald, P.G. Steg, J. Davidson, B. Hirshberg, et al., Saxagliptin and Cardiovascular Outcomes in Patients with Type 2 Diabetes Mellitus, N. Engl. J. Med. 369 (2013) 1317-1326. doi:10.1056/NEJMoa1307684.

[8] W.B. White, C.P. Cannon, S.R. Heller, S.E. Nissen, R.M. Bergenstal, G.L. Bakris, et al., Alogliptin after acute coronary syndrome in patients with type 2 diabetes., N. Engl. J. Med. 369 (2013) 1327-35. doi:10.1056/NEJMoa1305889.

[9] J.B. Green, M.A. Bethel, P.W. Armstrong, J.B. Buse, S.S. Engel, J. Garg, et al., Effect of Sitagliptin on Cardiovascular Outcomes in Type 2 Diabetes, N. Engl. J. Med. 373 (2015) 150608133014007. doi:10.1056/NEJMoa1501352. 
[10] M.A. Pfeffer, B. Claggett, R. Diaz, K. Dickstein, H.C. Gerstein, L. V Køber, et al., Lixisenatide in Patients with Type 2 Diabetes and Acute Coronary Syndrome., N. Engl. J. Med. 373 (2015) 2247-57. doi:10.1056/NEJMoa1509225.

[11] B. Zinman, C. Wanner, J.M. Lachin, D. Fitchett, E. Bluhmki, S. Hantel, et al., Empagliflozin, Cardiovascular Outcomes, and Mortality in Type 2 Diabetes, N. Engl. J. Med. 373 (2015) 150917085022006. doi:10.1056/NEJMoa1504720.

[12] M.P. Steven, D.H. Gilbert, B.-F. Kirstine, K. Peter, M.F. Johannes, N.A. Michael, et al., Liraglutide and Cardiovascular Outcomes in Type 2 Diabetes, N. Engl. J. Med. 375 (2016) 311-322. doi:10.1056/NEJMoa1603827.

[13] S.P. Marso, S.C. Bain, A. Consoli, F.G. Eliaschewitz, E. Jódar, L.A. Leiter, et al., Semaglutide and Cardiovascular Outcomes in Patients with Type 2 Diabetes, N. Engl. J. Med. (2016) NEJMoa1607141. doi:10.1056/NEJMoa1607141.

[14] P.G. Steg, R. Roussel, Randomized Trials to Evaluate Cardiovascular Safety of Antihyperglycemic Medications, Circulation. 134 (2016) 571-573.

doi:10.1161/CIRCULATIONAHA.116.021914. 